\title{
ON CENTRAL FUBINI-LIKE NUMBERS AND POLYNOMIALS
}

\author{
HACÈNE BELBACHIR AND YAHIA DJEMMADA
}

Received 15 January, 2019

\begin{abstract}
We introduce the central Fubini-like numbers and polynomials using Rota approach. Several identities and properties are established as generating functions, recurrences, explicit formulas, parity, asymptotics and determinantal representation.
\end{abstract}

2010 Mathematics Subject Classification: 05A10; 05A19; 05A40; 11B37; 11B73; 11B83; 11C08

Keywords: Fubini numbers and polynomials, central factorial numbers and polynomials, Fubinilike polynomials, difference operators, central difference

\section{INTRODUCTION}

We start by giving some definitions that will be used throughout this paper. For $n \geq 1$, the falling factorial denoted $x^{n}$ is defined by

$$
x^{\underline{n}}=x(x-1) \cdots(x-n+1),
$$

and the central factorial $x^{[n]}$, see $[4,9]$, is defined by

$$
x^{[n]}=x(x+n / 2-1)(x+n / 2-2) \cdots(x-n / 2+1) .
$$

We use the convention, $x^{0}=x^{[0]}=1$.

It is well-known that, for all non-negative integers $n$ and $k(k \leq n)$, Stirling numbers of the second kind are defined as the coefficients $S(n, k)$ in the expansion

$$
x^{n}=\sum_{k=0}^{n} S(n, k) x^{\underline{k}} .
$$

Riordan, in his book [15], shows that, for all non-negative integers $n$ and $k(k \leq n)$, the central factorial numbers of the second kind are the coefficients $T(n, k)$ in the expansion

$$
x^{n}=\sum_{k=0}^{n} T(n, k) x^{[k]} .
$$

In combinatorics, the number of ways to partition a set of $n$ elements into $k$ nonempty subsets are counted by Stirling numbers $S(n, k)$, and the central factorial numbers $T(2 n, 2 n-2 k)$ count the number of ways to place $k$ rooks on a 3D-triangle board of size $(n-1)$, see [11]. 


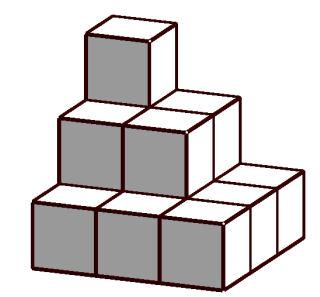

FIGURE 1. 3D-triangle board of size 3.

The coefficients $S(n, k)$ and $T(n, k)$ satisfy, respectively, the triangular recurrences

$$
S(n, k)=k S(n-1, k)+S(n-1, k-1) \quad(1 \leq k \leq n)
$$

and

$$
T(n, k)=\left(\frac{k}{2}\right)^{2} T(n-2, k)+T(n-2, k-2) \quad(2 \leq k \leq n),
$$

where

$$
S(n, k)=T(n, k)=0 \text { for } k>n, S(0,0)=T(0,0)=T(1,1)=1 \text { and } T(1,0)=0 .
$$

$S(n, k)$ and $T(n, k)$ admit also the explicit expressions

$$
\begin{gathered}
S(n, k)=\frac{1}{k !} \sum_{j=0}^{k}(-1)^{j}\left(\begin{array}{c}
k \\
j
\end{array}\right)(k-j)^{n}, \\
T(n, k)=\frac{1}{k !} \sum_{j=0}^{k}(-1)^{j}\left(\begin{array}{l}
k \\
j
\end{array}\right)\left(\frac{k}{2}-j\right)^{n} .
\end{gathered}
$$

\begin{tabular}{|c|ccccccc|}
\hline$n \backslash k$ & 0 & 1 & 2 & 3 & 4 & 5 & 6 \\
\hline 0 & 1 & & & & & & \\
1 & 0 & 1 & & & & & \\
2 & 0 & 1 & 1 & & & & \\
3 & 0 & 1 & 3 & 1 & & & \\
4 & 0 & 1 & 7 & 6 & 1 & & \\
5 & 0 & 1 & 15 & 25 & 10 & 1 & \\
6 & 0 & 1 & 31 & 90 & 65 & 15 & 1 \\
\hline
\end{tabular}

TABLE 1. The first few values of $S(n, k)$.

\begin{tabular}{|l|ccccccc|}
\hline & 0 & 1 & 2 & 3 & 4 & 5 & 6 \\
\hline 0 & 1 & & & & & & \\
1 & 0 & 1 & & & & & \\
2 & 0 & 0 & 1 & & & & \\
3 & 0 & $\frac{1}{4}$ & 0 & 1 & & & \\
4 & 0 & 0 & 1 & 0 & 1 & & \\
5 & 0 & $\frac{1}{16}$ & 0 & $\frac{5}{2}$ & 0 & 1 & \\
6 & 0 & 0 & 1 & 0 & 5 & 0 & 1 \\
\hline
\end{tabular}

TABLE 2. The first few values of $T(n, k)$. 
The usual difference operator $\Delta$, the shift operator $\mathrm{E}^{a}$ and the central difference operator $\delta$ are given respectively by

$$
\begin{gathered}
\Delta f(x)=f(x+1)-f(x), \\
\mathrm{E}^{a} f(x)=f(x+a)
\end{gathered}
$$

and

$$
\delta f(x)=f(x+1 / 2)-f(x-1 / 2) .
$$

Riordan, [15], mentioned that the central factorial operator $\delta$ satisfies the following property

$$
\delta f_{n}(x)=n f_{n-1}(x)
$$

where $\left(f_{n}(x)\right)_{n \geq 0}$ is a sequence of polynomials with $f_{0}(x)=1$.

We can also express $\delta$ by means of both $\Delta$ and $\mathrm{E}^{a}$, see [9,15], as follows:

$$
\delta f(x)=\Delta \mathrm{E}^{-1 / 2} f(x) .
$$

For more details about difference operators, we refer the reader to [9].

\section{Central Fubini-LiKe numbers AND POlynomials}

In 1975, Tanny [17], introduced the Fubini polynomials (or ordered Bell polynomials) $F_{n}(x)$ by applying a linear transformation $\mathcal{L}$ defined as

$$
\mathcal{L}\left(x^{n}\right):=n ! x^{n} .
$$

The polynomials $F_{n}(x)$ are given by

$$
F_{n}(x):=\sum_{k \geq 0}^{n} k ! S(n, k) x^{k},
$$

according to,

$$
F_{n}(x):=\mathcal{L}\left(x^{n}\right)=\mathcal{L}\left(\sum_{k=0}^{n} S(n, k) x^{\underline{k}}\right)=\sum_{k=0}^{n} S(n, k) \mathcal{L}\left(x^{\underline{k}}\right)=\sum_{k=0}^{n} k ! S(n, k) x^{k} .
$$

Putting $x=1$ in (2) we get

$$
F_{n}:=F_{n}(1)=\sum_{k=0}^{n} k ! S(n, k),
$$

which is the $n$-th Fubini number.

The Fubini polynomial $F_{n}(x)$ has the exponential generating function given by, see [17],

$$
\sum_{n=0} F_{n}(x) \frac{t^{n}}{n !}=\frac{1}{1-x\left(e^{t}-1\right)} .
$$

For more details concerning Fubini numbers and polynomials, see [3, 6, 8, 12, 17, 18, 20] and papers cited therein.

Now, we introduce the linear transformation $Z$ as follows. 
Definition 1. For $n \geq 0$, we define the transformation

$$
z\left(x^{[n]}\right)=n ! x^{n} .
$$

Then, we have

$$
Z\left(x^{n}\right)=Z\left(\sum_{k=0}^{n} T(n, k) x^{[k]}\right)=\sum_{k=0}^{n} T(n, k) Z\left(x^{[k]}\right)=\sum_{k=0}^{n} k ! T(n, k) x^{k} .
$$

And due to Formula (1.6), we are now able to introduce the main notion of the present paper.

Definition 2. The $n$-th central Fubini-like polynomial is given by

$$
\mathfrak{C}_{n}(x):=\sum_{k=0}^{n} k ! T(n, k) x^{k} .
$$

Setting $x=1$, we obtain the central Fubini-like numbers,

$$
\mathfrak{C}_{n}=\mathfrak{C}_{n}(1):=\sum_{k=0}^{n} k ! T(n, k) .
$$

The first central polynomials $\mathfrak{C}_{n}(x)$ are given in Table 3.

\begin{tabular}{|l|l|l|}
\hline$n$ & $\mathfrak{C}_{2 n}(x)$ & $2^{2 n} \mathbb{C}_{2 n+1}(x)$ \\
\hline 0 & 1 & $x$ \\
\hline 1 & $2 x^{2}$ & $x+24 x^{3}$ \\
\hline 2 & $2 x^{2}+24 x^{4}$ & $x+240 x^{3}+1920 x^{5}$ \\
\hline 3 & $2 x^{2}+120 x^{4}+720 x^{6}$ & $x+2184 x^{3}+67200 x^{5}+322560 x^{7}$ \\
\hline 4 & $2 x^{2}+504 x^{4}+10080 x^{6}+40320 x^{8}$ & $x+19680 x^{3}+1854720 x^{5}+27095040 x^{7}+92897280 x^{9}$ \\
\hline
\end{tabular}

TABLE 3. First value of $\mathfrak{C}_{n}(x)$.

The first few central Fubini-like numbers are

$$
\begin{array}{ll}
\left(\mathfrak{C}_{2 n}\right)_{n \geq 0}: & 1,2,26,842,50906,4946282,704888186,138502957322, \ldots \\
\left(2^{2 n} \mathfrak{C}_{2 n+1}\right)_{n \geq 0}: & 1,25,2161,391945,121866721,57890223865,38999338931281, \ldots
\end{array}
$$

\subsection{Exponential generating function}

We begin by establishing the exponential generating function of the central Fubinilike polynomials.

Theorem 1. The polynomials $\mathfrak{\mathfrak { C }}_{n}(x)$ have the following exponential generating function

$$
G(x ; t):=\sum_{n=0} \mathfrak{C}_{n}(x) \frac{t^{n}}{n !}=\frac{1}{1-2 x \sinh (t / 2)} .
$$


Proof. We have

$$
\sum_{n=0} \mathfrak{C}_{n}(x) \frac{t^{n}}{n !}=\sum_{n=0}^{\infty} \sum_{k=0}^{n} k ! T(n, k) x^{k} \frac{t^{n}}{n !}=\sum_{k=0} k ! x^{k} \sum_{n=k}^{\infty} T(n, k) \frac{t^{n}}{n !},
$$

from [15, p. 214], we have

$$
\sum_{n=0} T(n, k) \frac{t^{n}}{n !}=\frac{1}{k !}(2 \sinh (t / 2))^{k}
$$

therefore

$$
\sum_{n=0} \mathfrak{C}_{n}(x) \frac{t^{n}}{n !}=\sum_{k=0}^{\infty}(2 \sinh (t / 2))^{k} x^{k}=\frac{1}{1-2 x \sinh (t / 2)}
$$

Corollary 1. The sequence $\left(\mathfrak{C}_{n}\right)_{n \geq 0}$ has the following exponential generating function

$$
\sum_{k=0}^{n} \mathfrak{C}_{n} \frac{t^{n}}{n !}=\frac{1}{1-2 \sinh (t / 2)}
$$

\subsection{Explicit representations}

In this subsection we propose some explicit formulas for the central Fubini-like polynomials, we start by the derivative representation.

Proposition 1. The polynomials $\left(\mathfrak{C}_{n}(x)\right)_{n \geq 0}$ correspond to the higher derivative expression

$$
\mathfrak{C}_{n}(x)=\left.\sum_{k=0}^{\infty} \frac{\partial^{n}}{\partial^{n} t}(2 x \sinh (t / 2))^{k}\right|_{t=0} .
$$

Proof. Let

$\left.\frac{\partial^{n}}{\partial^{n} t}\left(\sum_{m=0}^{\infty} \mathfrak{C}_{m}(x) \frac{t^{m}}{m !}\right)\right|_{t=0}=\left.\sum_{m=n}^{\infty} \mathfrak{C}_{m}(x) \frac{t^{m-n}}{(m-n) !}\right|_{t=0}=\left.\sum_{m=0}^{\infty} \mathfrak{C}_{n+m}(x) \frac{t^{m}}{m !}\right|_{t=0}=\mathfrak{C}_{n}(x)$.

Thus from Theorem 1 we get the result.

From Formula (1.6), it is clear that the following proposition holds.

Proposition 2. The central Fubini-like polynomials satisfy the following explicit formula

$$
\mathfrak{C}_{n}(x)=\sum_{k=0}^{n} x^{k} \sum_{j=0}^{k}(-1)^{j}\left(\begin{array}{l}
k \\
j
\end{array}\right)(k / 2-j)^{n} .
$$

Proof. It suffices to replace $T(n, k)$ in Equation (2.6) by its explicit formula (Equation (1.6)),

$$
\mathfrak{C}_{n}(x)=\sum_{k=0}^{n} k ! T(n, k) x^{k}=\sum_{k=0}^{n} x^{k} \sum_{j=0}^{k}(-1)^{j}\left(\begin{array}{l}
k \\
j
\end{array}\right)(k / 2-j)^{n} .
$$


Theorem 2. For non-negative $n$, the following explicit representation holds true.

$$
\mathfrak{C}_{n}(x)=x \sum_{k=0}^{n-1}\left(\begin{array}{l}
n \\
k
\end{array}\right) \sum_{j=0}^{k}\left(\begin{array}{l}
k \\
j
\end{array}\right)\left(\frac{-1}{2}\right)^{k-j} \mathfrak{C}_{j}(x)=x \sum_{j=0}^{n-1}\left(\begin{array}{l}
n \\
j
\end{array}\right) \delta\left[0^{n-j}\right] \mathfrak{C}_{j}(x),
$$

where $\delta\left[0^{n-j}\right]=(1 / 2)^{n-j}-(-1 / 2)^{n-j}$.

The proof will depend on Lemma 1, Lemma 2 and Relation (1.8).

Lemma 1. For all polynomials $p_{n}(x)$ the following relation holds true.

$$
Z\left(p_{n}(x)\right)=x Z\left(\delta p_{n}(x)\right) \text {. }
$$

Proof. We have

$$
Z\left(x^{[n]}\right)=n ! x^{n}=x n(n-1) ! x^{n-1}=x Z\left(n x^{[n-1]}\right)=x Z\left(\delta x^{[n]}\right),
$$

as any polynomial can be written as sums of central factorials $x^{[n]}$. Thus, we have the result.

Lemma 2 (Tanny [17]). For all polynomials $p_{n}(x)$ we have

$$
\Delta p_{n}(x)=\sum_{k=0}^{n-1}\left(\begin{array}{l}
n \\
k
\end{array}\right) p_{k}(x)
$$

Now we give the proof of Theorem 2,

Proof of Theorem 2. Using Lemma 1, Lemma 2 and setting $p_{n}(x)=x^{n}$, we get

$$
\begin{aligned}
Z\left(x^{n}\right) & =x Z\left(\delta x^{n}\right)=x Z\left(\Delta E^{-1 / 2} x^{n}\right)=x Z\left(\Delta\left(x-\frac{1}{2}\right)^{n}\right) \\
& =x z\left(\sum_{k=0}^{n-1}\left(\begin{array}{l}
n \\
k
\end{array}\right)\left(x-\frac{1}{2}\right)^{k}\right)=x z\left(\sum_{k=0}^{n-1}\left(\begin{array}{l}
n \\
k
\end{array}\right) \sum_{j=0}^{k}\left(\begin{array}{l}
k \\
j
\end{array}\right)\left(\frac{-1}{2}\right)^{k-j} x^{j}\right) \\
& =x \sum_{k=0}^{n-1}\left(\begin{array}{l}
n \\
k
\end{array}\right) \sum_{j=0}^{k}\left(\begin{array}{l}
k \\
j
\end{array}\right)\left(\frac{-1}{2}\right)^{k-j} \mathfrak{c}_{j}(x) .
\end{aligned}
$$

Using binomial product identity $\left(\begin{array}{l}n \\ k\end{array}\right)\left(\begin{array}{l}k \\ j\end{array}\right)=\left(\begin{array}{l}n-j \\ k-j\end{array}\right)\left(\begin{array}{l}n \\ j\end{array}\right)$, we get the result.

Corollary 2. The central Fubini-like numbers satisfy

$$
\mathfrak{c}_{n}=\sum_{j=0}^{n-1}\left(\begin{array}{l}
n \\
j
\end{array}\right) \delta\left[0^{n-j}\right] \mathfrak{c}_{j}
$$

Now we give an explicit formula connecting the central Fubini-like polynomials with Stirling numbers of the second kind $S(n, k)$, 
Theorem 3. The central Fubini-like polynomials $\mathfrak{\mathfrak { C }}_{n}(x)$ satisfy

$$
\mathfrak{C}_{n}(x)=\sum_{k=0}^{n} k ! x^{k} \sum_{j=0}^{n}\left(\begin{array}{l}
n \\
j
\end{array}\right)\left(\frac{-k}{2}\right)^{j} S(n-j, k) .
$$

Proof. From Theorem 1, we have

$$
\sum_{n=0} \mathfrak{c}_{n}(x) \frac{t^{n}}{n}=\frac{1}{1-2 x \sinh (t / 2)} .
$$

Using the exponential form of $2 x \sinh (t / 2)$ we get

$$
\sum_{n=0} \mathfrak{C}_{n}(x) \frac{t^{n}}{n}=\frac{1}{1-x e^{(-t / 2)}\left(e^{t}-1\right)}=\sum_{k=0} x^{k} e^{(-k t / 2)}\left(e^{t}-1\right)^{k} .
$$

It is also known that

$$
\sum_{n=0} S(n, k) \frac{t^{n}}{n !}=\frac{\left(e^{t}-1\right)^{k}}{k !}
$$

Therefore

$$
\sum_{n=0} \mathfrak{C}_{n}(x) \frac{t^{n}}{n}=\sum_{k=0} x^{k} k ! \sum_{n=0}\left(\frac{-k}{2}\right)^{n} \frac{t^{n}}{n !} \sum_{n=0} S(n, k) \frac{t^{n}}{n !} .
$$

Then Cauchy's product implies the identity.

Corollary 3. The central Fubini-like numbers $\mathfrak{C}_{n}$ satisfy

$$
\mathfrak{C}_{n}=\sum_{k=0}^{n} k ! \sum_{j=0}^{n}\left(\begin{array}{c}
n \\
j
\end{array}\right)\left(\frac{-k}{2}\right)^{j} S(n-j, k) .
$$

\subsection{Umbral representation}

Umbral (or Blissard or symbolic) calculus originated as a method for discovering and proving combinatorial identities in which subscripts are treated as powers. Bell in [1] gave a postulational bases of this calculus. In this section we use the following property given by Riordan [16]. As specified by the author in [16], "A sequence $a_{0}, a_{1}, \ldots$ may be replaced by $a^{0}, a^{1}, \ldots$ with the exponents are treated as powers during all formal operations, and only restored as indexes when operations are completed". Then when we have

$$
a_{n}=\sum_{k=0}\left(\begin{array}{l}
n \\
k
\end{array}\right) b_{k} c_{n-k}
$$

we can write it as

$$
a_{n}=(b+c)^{n},
$$

where $b^{n} \equiv b_{n}$ and $c^{n} \equiv c_{n}$. We note that $b^{0}$ and $c^{0}$ is not necessary equal to 1 .

In the following theorem we use the umbral notation $\mathfrak{C}_{k}(x) \equiv \mathfrak{C}^{k}(x)$ and $\mathfrak{C}_{k} \equiv \mathfrak{C}^{k}$.

Theorem 4. Let $n$ be a non-negative integer, for all real $x$ we have

$$
\mathfrak{C}_{n}(x)=x\left[(\mathfrak{C}(x)+1 / 2)^{n}-(\mathfrak{C}(x)-1 / 2)^{n}\right] .
$$


Proof. From Theorem 2 and using the umbral notation, a simple calculation gives the umbral representation result.

Corollary 4. For non-negative integer $n$, we have

$$
\mathfrak{C}_{n}=(\mathfrak{C}+1 / 2)^{n}-(\mathfrak{C}-1 / 2)^{n} .
$$

\subsection{Parity}

A function $f(x)$ is said to be even when $f(x)=f(-x)$ for all $x$ and it is said to be odd when $f(x)=-f(-x)$.

Theorem 5. For all non-negative $n$ and real variable $x$ we have

$$
\mathfrak{C}_{n}(x)=(-1)^{n} \mathfrak{C}_{n}(-x) .
$$

Proof. Using the fact that the function $f: t \mapsto \sinh (t)$ is odd, this gives $G(x ; t)=$ $G(-x ;-t)$, then comparing the coefficients of $t^{n} / n !$ in $G(x ; t)$ and $G(-x ;-t)$ the theorem follows.

Corollary 5. The polynomials $\mathfrak{C}_{n}(x)$ are odd if and only if $n$ is odd.

Proof. Using Theorem 5, it suffices to replace $n$ by $2 k+1$ (resp. $2 k$ ) and establish the property.

\subsection{Recurrences and derivatives of higher order}

Now we are interested to derive some recurrences for $\mathbb{C}_{n}(x)$ in terms of their derivatives.

First, we deal with a recurrence of second order.

Theorem 6. For $n \geq 2$, the polynomials $\mathfrak{C}_{n}(x)$ satisfy the following recurrence relation

$$
\mathfrak{C}_{n}(x)=2 x^{2} \mathfrak{C}_{n-2}(x)+\left(\frac{x}{4}+4 x^{3}\right) \mathfrak{C}_{n-2}^{\prime}(x)+\left(\frac{x^{2}}{4}+x^{4}\right) \mathfrak{C}_{n-2}^{\prime \prime}(x) .
$$

Here $\mathfrak{C}_{n}^{\prime}(x)$ and $\mathbb{C}_{n}^{\prime \prime}(x)$ are respectively the first and second derivative of $\mathfrak{C}_{n}(x)$.

Proof. From Equation (1.4) we have

$$
\begin{aligned}
\mathfrak{C}_{n}(x) & =\sum_{k=0}^{n} k ! T(n, k) x^{k} \\
& =\sum_{k=2}^{n} k ! T(n-2, k-2) x^{k}+\frac{1}{4} \sum_{k=0}^{n} k^{2} k ! T(n-2, k) x^{k} \\
& =\sum_{k=0}^{n}(k+2) ! T(n-2, k) x^{k+2}+\frac{x}{4}\left(\sum_{k=0}^{n} k k ! T(n-2, k) x^{k}\right)^{\prime}
\end{aligned}
$$




$$
\begin{aligned}
& =x^{2}\left(x^{2} \sum_{k=0}^{n} k ! T(n-2, k) x^{k}\right)^{\prime \prime}+\frac{x}{4}\left(x\left(\sum_{k=0}^{n} k ! T(n-2, k) x^{k}\right)^{\prime}\right)^{\prime} \\
& =x^{2}\left(x^{2} \mathbb{C}_{n-2}(x)\right)^{\prime \prime}+\frac{x}{4}\left(x \mathfrak{C}_{n-2}^{\prime}(x)\right)^{\prime} \\
& =2 x^{2} \mathbb{C}_{n-2}(x)+\left(\frac{x}{4}+4 x^{3}\right) \mathfrak{C}_{n-2}^{\prime}(x)+\left(\frac{x^{2}}{4}+x^{4}\right) \mathfrak{C}_{n-2}^{\prime \prime}(x),
\end{aligned}
$$

this concludes the proof.

In the next theorem we give a recurrence formula for the $r$-th derivative of $\mathfrak{C}_{n}(x)$.

Proposition 3. The r-th derivative of $G(x ; t)$, defined in (2.8), is given by

$$
\frac{\partial^{r}}{\partial^{r} x} G(x ; t)=\frac{r !}{x^{r}} G(x ; t)(G(x ; t)-1)^{r} .
$$

Proof. Induction on $r$ implies the equality.

Theorem 7. Let $\mathfrak{C}_{n}^{(r)}(x)$ be the r-th derivative of $\mathfrak{C}_{n}(x)$. Then $\mathfrak{C}_{n}^{(r)}(x)$ is given by

$$
\mathfrak{C}_{n}^{(r)}(x)=\frac{r !}{x^{r}} \sum_{k=0}^{r}\left(\begin{array}{l}
r \\
k
\end{array}\right)(-1)^{r-k} \sum_{j_{0}+j_{1}+\cdots+j_{k}=n}\left(\begin{array}{c}
n \\
j_{0}, j_{1}, \ldots, j_{k}
\end{array}\right) \mathfrak{C}_{j_{0}}(x) \mathfrak{C}_{j_{1}}(x) \cdots \mathfrak{C}_{j_{k}}(x) .
$$

Proof. Using Proposition 3, by applying Cauchy product and comparing the coefficients of $t^{n} / n !$, we get the result.

Corollary 6. The following equality holds for any real $x$ :

$$
x \mathfrak{C}_{n}^{\prime}(x)=\sum_{k=0}^{n-1}\left(\begin{array}{l}
n \\
k
\end{array}\right) \mathbb{C}_{k}(x) \mathfrak{C}_{n-k}(x) .
$$

Proof. Setting $r=1$ in Proposition 3, we get the first derivative of $G(x ; t)$ as

$$
\begin{aligned}
\frac{\partial}{\partial x} G(x ; t) & =\frac{2 \sinh \left(\frac{t}{2}\right)}{\left(1-2 x \sinh \left(\frac{t}{2}\right)\right)^{2}}=\frac{G(x ; t)}{x}(G(x ; t)-1), \\
x \frac{\partial}{\partial x} G(x ; t) & =G(x ; t)^{2}-G(x ; t), \\
x \sum_{n=0} \mathfrak{C}_{n}^{\prime}(x) \frac{t^{n}}{n !} & =\left(\sum_{n=0} \mathfrak{C}_{n}(x) \frac{t^{n}}{n !}\right)^{2}-\sum_{n=0} \mathfrak{C}_{n}(x) \frac{t^{n}}{n !}
\end{aligned}
$$

then applying the Cauchy product in the right hand side and comparing the coefficients of $t^{n} / n$ ! we get the result. 


\subsection{Integral representation}

Integral representation is a fundamental property in analytic combinatorics. The central Fubini-like polynomials can be represented as well.

Theorem 8. The polynomials $\mathfrak{C}_{n}(x)$ satisfy

$$
\mathfrak{C}_{n}(x)=\frac{2 n !}{\pi} \mathbf{I m} \int_{0}^{\pi} \frac{\sin (n \theta)}{1-2 x \sinh \left(e^{i \theta} / 2\right)} \partial \theta .
$$

Proof. We will use here the known identity, see [5],

$$
k^{n}=\frac{2 n !}{\pi} \mathbf{I m} \int_{0}^{\pi} \exp \left(k e^{i \theta}\right) \sin (n \theta) \partial \theta .
$$

We have

$$
\begin{aligned}
\mathfrak{C}_{n}(x) & =\sum_{k=0}^{\infty} k ! T(n, k) x^{k} \\
& =\sum_{k=0}^{\infty} x^{k} \sum_{j=0}^{k}(-1)^{j}\left(\begin{array}{c}
k \\
j
\end{array}\right)\left(\frac{k}{2}-j\right)^{n} \\
& =\sum_{k=0}^{\infty} x^{k} \sum_{j=0}^{k}(-1)^{j}\left(\begin{array}{c}
k \\
j
\end{array}\right) \frac{2 n !}{\pi} \mathbf{I m} \int_{0}^{\pi} \exp \left((k / 2-j) e^{i \theta}\right) \sin (n \theta) \partial \theta \\
& =\frac{2 n !}{\pi} \mathbf{I m} \int_{0}^{\pi} \sin (n \theta) \sum_{k=0}^{\infty} x^{k} \exp \left(-\frac{k}{2} e^{i \theta}\right)\left(\exp \left(e^{i \theta}\right)-1\right)^{k} \partial \theta \\
& =\frac{2 n !}{\pi} \mathbf{I m} \int_{0}^{\pi} \frac{\sin (n \theta)}{1-2 x \sinh \left(e^{i \theta} / 2\right)} \partial \theta .
\end{aligned}
$$

\subsection{Determinantal representation}

Several papers have been published on determinantal representations of many sequences as Bernoulli numbers, Euler numbers, ordered Bell numbers (or Fubini numbers), etc.

Komatsu and Ramírez in a recent paper gives the following theorem.

Theorem 9 (Komatsu \& Ramírez [10]). Let $(R(j))_{j \geq 0}$ be a sequence, and let $\alpha_{n}$ be defined by the following determinantal expression for all $n \geq 1$ :

$$
\alpha_{n}=\left|\begin{array}{ccccc}
R(1) & 1 & & & \\
R(2) & R(1) & & & \\
\vdots & \vdots & \ddots & 1 & \\
R(n-1) & R(n-2) & \cdots & R(1) & 1 \\
R(n) & R(n-1) & \cdots & R(2) & R(1)
\end{array}\right|
$$


Then we have

$$
\alpha_{n}=\sum_{j=1}^{n}(-1)^{j-1} R(j) \alpha_{n-j} \quad(n \geq 1)
$$

We set $\alpha_{0}=1$.

By applying the previous theorem we get

Theorem 10. For $n \geq 1$, we have

$$
\frac{\mathfrak{c}_{n}(x)}{n !}=\left|\begin{array}{ccccc}
R(1) & 1 & & & \\
R(2) & R(1) & & & \\
\vdots & \vdots & \ddots & 1 & \\
R(n-1) & R(n-2) & \cdots & R(1) & 1 \\
R(n) & R(n-1) & \cdots & R(2) & R(1)
\end{array}\right|
$$

where

$$
R(j)=x \frac{(-1)^{j-1}}{j !} \delta\left[0^{j}\right]=x \frac{(-1)^{j-1}}{j !}\left(\left(\frac{1}{2}\right)^{j}-\left(-\frac{1}{2}\right)^{j}\right) .
$$

Proof. From Theorem 2 we have,

$$
\begin{aligned}
& \mathfrak{C}_{n}(x)=x \sum_{j=0}^{n-1}\left(\begin{array}{l}
n \\
j
\end{array}\right) \delta\left[0^{n-j}\right] \mathfrak{C}_{j}(x)=x \sum_{j=1}^{n}\left(\begin{array}{l}
n \\
j
\end{array}\right) \delta\left[0^{j}\right] \mathfrak{C}_{n-j}(x) \\
& \frac{\mathfrak{C}_{n}(x)}{n !}=\sum_{j=1}^{n} \frac{x}{j !} \delta\left[0^{j}\right] \frac{\mathfrak{C}_{n-j}(x)}{(n-j) !} .
\end{aligned}
$$

It suffices to set $\alpha_{n}=\frac{\mathfrak{c}_{n}(x)}{n !}$ and $R(j)=x \frac{(-1)^{j-1}}{j !} \delta\left[0^{j}\right]$ to get the result.

Remark 1. The function $R(j)=0$ for $j$ even.

Using Remark 1, we establish the following binomial convolution for the polynomials $\mathfrak{C}_{n}(x)$.

Theorem 11. For $n \geq 0$ we have

$$
\mathfrak{C}_{n+1}(x)=x \sum_{k=0}^{\lfloor n / 2\rfloor} 4^{-k}\left(\begin{array}{c}
n+1 \\
2 k+1
\end{array}\right) \mathfrak{C}_{n-2 k}(x)
$$

Proof. From Remark 1 and using Formula (2.16) with $\alpha_{n}=\mathbb{C}_{n}(x) / n$ ! and $R(j)=x \frac{(-1)^{j-1}}{j !}\left(\left(\frac{1}{2}\right)^{j}-\left(-\frac{1}{2}\right)^{j}\right)$ we get the result.

Remark 2. Formula (2.18) is better than result of Theorem 2 from a computational point of view. 


\subsection{Asymptotic result with respect to $\mathfrak{C}_{n}$}

Find an asymptotic behaviour of a sequence $\left(a_{n}\right)_{n \geq 0}$ means to find a second function depending on $n$ simple than the expression of $a_{n}$ which gives a good approximation to the values of $a_{n}$ when $n$ is large.

In this subsection, we are interested to obtaining the asymptotic behaviour of the central Fubini-like numbers.

Let $\left(a_{n}\right)_{n \geq 0}$ be a sequence of non-negative real numbers, the asymptotic behaviour $a_{n}$ is closely tied to the poles in $G(z)$, where $G(z)$ is the generating function of $a_{n}$,

$$
G(z)=\sum_{n=0} a_{n} z^{n}
$$

Wilf, in his book [19] and Flajolet et al. in [7] gave a method to determine the asymptotic behaviour $a_{n}$ which can be summarized in the following steps:

(1) Find the poles $z_{0}, z_{1}, \ldots, z_{s}$ in $G(z)$.

(2) Calculate the principal parts $P\left(G(z), z_{i}\right)$ at the dominant singularities $z_{i}$ (which have the smallest modulus $R$ ) as

$$
P\left(G(z), z_{i}\right)=\frac{\operatorname{Res}\left(G(z), z_{i}\right)}{\left(z-z_{i}\right)},
$$

where $\operatorname{Res}\left(G(z), z_{i}\right)$ is the residue of $G(z)$ at the pole $z_{i}$.

(3) Set $H(z)=\sum_{i=0}^{s} P\left(G(z), z_{i}\right)$ then write $H(z)$ as the expansion below,

$$
H(z)=\sum_{n=0} b_{n} z^{n}
$$

(4) The sequence $\left(b_{n}\right)_{n=0}$ is the asymptotic behaviour of $a_{n}$ when $n$ is big enough,

$$
a_{n} \sim b_{n}+O\left(\left(\frac{1}{R^{\prime}}+\varepsilon\right)^{n}\right), \quad n \longmapsto \infty .
$$

where $R^{\prime}$ is the next smallest modulus of the poles.

For more details about singularities analysis method we refer to [7].

Remark 3. Poles $z_{0}, z_{1}, \ldots, z_{s}$ are considered as simple poles (has a multiplicity equal to 1).

Analytic methods of determining the asymptotic behavior of a sequence $\left(a_{n}\right)_{n}$ are widely discussed on $[2,7,13,14,19]$.

Theorem 12. The asymptotic behaviour of the $\mathfrak{C}_{n}$ is given by

$$
\mathfrak{C}_{n} \sim \frac{n !}{2^{n} \sqrt{5} \log ^{n+1}(\phi)}+O\left((0.15732+\varepsilon)^{n}\right), \quad n \longmapsto \infty
$$

where $\phi$ is the Golden ratio.

Proof. Applying the previous steps in the generating function $G(z)=\frac{1}{1-2 \sinh (z / 2)}$ gives 
(1) The poles of $G(z)$ are

$$
z_{0}=-2 \log \left(\frac{1+\sqrt{5}}{2}\right)+2 i \pi+4 i \pi k \text { and } z_{1}=2 \log \left(\frac{1+\sqrt{5}}{2}\right)+4 i \pi k,
$$

with $k \in \mathbb{Z}$.

(2) By setting $k=0$, the dominant singularity is $z_{1}=2 \log (\phi)$ (the modulus $R=$ 0.96), then,

$$
P\left(G(z), z_{1}\right)=-\frac{2}{\sqrt{5}(z-2 \log (\phi))} .
$$

(3) Set $H(z)=-\frac{2}{\sqrt{5}(z-2 \log (\phi))}$, if we write $H(z)$ as the expansion we get

$$
H(z)=\sum_{n=0} \frac{1}{2^{n} \sqrt{5} \log ^{n+1}(\phi)} z^{n} .
$$

(4) The the next smallest modulus of the poles $R^{\prime}=6.356 \ldots$, then the asymptotic behaviour of $\mathfrak{C}_{n}$ when $n$ is big enough is,

$$
\mathfrak{C}_{n} \sim \frac{n !}{2^{n} \sqrt{5} \log ^{n+1}(\phi)}+O\left((0.15732+\varepsilon)^{n}\right), \quad n \longmapsto \infty .
$$

\section{ACKNOWLEDGMENTS}

We would like to thank the anonymous reviewers for their suggestions and comments. We would also like to thank Benjamin Hackl from University of Klagenfurt, Austria, for helpful discussions.

\section{REFERENCES}

[1] E. T. Bell, "Postulational bases for the umbral calculus," American Journal of Mathematics, vol. 62 , no. 1, pp. 717-724, 1940, doi: 10.2307/2371481.

[2] E. A. Bender, "Asymptotic methods in enumeration," SIAM review, vol. 16, no. 4, pp. 485-515, 1974, doi: 10.1137/1016082.

[3] K. N. Boyadzhiev, "A series transformation formula and related polynomials," International Journal of Mathematics and Mathematical Sciences, vol. 2005, no. 23, pp. 3849-3866, 2005, doi: 10.1155/IJMMS.2005.3849.

[4] P. L. Butzer, K. Schmidt, E. Stark, and L. Vogt, "Central factorial numbers; their main properties and some applications." Numerical Functional Analysis and Optimization, vol. 10, no. 5-6, pp. 419-488, 1989, doi: 10.1080/01630568908816313.

[5] D. Callan, "Cesaro's integral formula for the Bell numbers (corrected)," arXiv preprint arXiv:0708.3301, 2007.

[6] A. Dil and V. Kurt, "Investigating geometric and exponential polynomials with Euler-Seidel matrices," J. Integer Seq, vol. 14, no. 4, 2011.

[7] P. Flajolet and R. Sedgewick, Analytic combinatorics. Cambridge University Press, 2009.

[8] O. A. Gross, "Preferential arrangements," The American Mathematical Monthly, vol. 69, no. 1, pp. 4-8, 1962, doi: 10.1080/00029890.1962.11989826. 
[9] C. Jordan and K. Jordán, Calculus of finite differences. American Mathematical Soc., 1965, vol. 33.

[10] T. Komatsu and J. L. Ramírez, "Some determinants involving incomplete Fubini numbers," An. $S_{s}$ tiint. Univ. "Ovidius" Constant $t_{s}$ S Ser. Mat. 26, no.3, 2018, doi: 10.2478/auom-2018-0038.

[11] N. Krzywonos and F. Alayont, "Rook polynomials in three and higher dimensions," Involve, vol. 6, no. 1, pp. 35-52, 2013, doi: 10.2140/involve.2013.6.35.

[12] I. Mezô, "Periodicity of the last digits of some combinatorial sequences," J. Integer Seq, vol. 17, pp. 1-18, 2014.

[13] A. M. Odlyzko, "Asymptotic enumeration methods," Handbook of combinatorics, vol. 2, no. 1063, p. $1229,1995$.

[14] J. Plotkin and J. Rosenthal, "Some asymptotic methods in combinatorics," Journal of the Australian Mathematical Society, vol. 28, no. 4, pp. 452-460, 1979, doi: 10.1017/S1446788700012593.

[15] J. Riordan, Combinatorial identities. Wiley New York, 1968, vol. 6.

[16] J. Riordan, Introduction to combinatorial analysis. C Courier Corporation, 2012.

[17] S. M. Tanny, "On some numbers related to the Bell numbers," Canadian Mathematical Bulletin, vol. 17, no. 5, pp. 733-738, 1975, doi: 10.4153/CMB-1974-132-8.

[18] W. A. Whitworth, Choice and chance: with 1000 exercises. D. Bell and Company;[etc., etc.,], 1901.

[19] H. S. Wilf, generatingfunctionology. AK Peters/CRC Press, 2005.

[20] D. Zeitlin, "Remarks on a formula for preferential arrangements," The American Mathematical Monthly, vol. 70, no. 2, pp. 183-187, 1963, doi: 10.2307/2312890.

Authors' addresses

Hacène Belbachir

USTHB, Faculty of Mathematics, RECITS Laboratory, BP 32, El Alia, 16111, Bab Ezzouar, Algiers, Algeria

E-mail address: hacenebelbachiregmail.com, hbelbachir@usthb.dz

Yahia Djemmada

USTHB, Faculty of Mathematics, RECITS Laboratory, BP 32, El Alia, 16111, Bab Ezzouar, Algiers, Algeria

E-mail address: yahia.djem@gmail.com, ydjemmada@usthb.dz 